ด

columns
Department of General Practice. This focuses on clinical teaching skills of explanation, effective questioning, delivering feedback, bedside teaching techniques student assessment and evaluation of teaching. Given the effect that undergraduate psychiatry teaching may have on subsequent career choice (Brockington \& Mumford, 2002), it could be argued that improving the teaching skills of our trainees will pay dividends for recruitment into psychiatry.

BROCKINGTON, I. \& MUMFORD, D. B. (2002) Recruitment into psychiatry. British Journal of Psychiatry, 180, 307-312.

Camilla Langan Clinical Lecturer in Psychiatry, Department of Psychiatry, Clinical Science Institute, National University of Ireland, Galway, Ireland, email: camilla.langan@nuigalway.ie

doi: 10.1192/pb.31.7.273d

\section{Have you got a right please?}

The emphasis on the proposed health bil is to protect workers and the general public from second-hand smoke. Passive smoking causes about 12000 deaths per year (Royal College of Physicians, 2005); 500 of these are due to exposure at work.

As my workplace extends to patients' homes, should I not expect the same protection as I would in an NHS building and therefore demand that a patient ceases to smoke in their own home? Community doctors and nurses, who spend vast amounts of time in patients' homes would argue that the amount of second-hand smoke inhaled per day is sometimes very high. Many of us have been in the situation where we battle through a smog of smoke just to find the patient. The next hour is painful, every breath a chore, until we hear a polyphonic wheeze deep inside our own struggling lungs. We leave and take our first heavenly gasp of fresh air, but every breath for the remainder of the day is tainted by the smell of ashtray clinging to our clothes.

Pregnant workers will understandably go to great lengths to avoid cigarette smoke and subsequent harm to their baby. Is it not their right, and some might say the right of the unborn child, to refuse to enter the house of a patient who smokes?

Of course, it is unrealistic to expect patients to stop smoking in their own homes. We could, however, follow our friends in the health visitor sector who have been requesting for over a year that patients do not smoke for an hour prior to their visit. If this practice is recognised as a condition of the visit, by previous written request, it gives health workers the right to refuse to enter the home if this is not adhered to.
Some would say that asking patients not to smoke in their own homes goes too far, adding to the 'Big Brother' milieu in which we find ourselves. Others would say that the culprits' human rights appear to be more valuable than those of the innocents, and that these rights sometimes outweigh reason. Our needs are important and we should enforce a onehour smoking ban.

ROYAL COLLEGE OF PHYSICIANS (2005) Going Smoke-Free: The Medical Case for Clean Air in the Home, at Work and in Public Places. Royal College of Physicians.

Matthew Cahill Specialist Registrar in Psychiatry East London Rotation

doi: 10.1192/pb.31.7.274

\section{Payment for medication}

Ethical deliberations aside, bribing patients with cash to accept depot medication clearly (and perhaps fatally) contradicts the message that the medication is a worthwhile and positive offering in itself Moreover, it cheapens and demeans the receiver who becomes one whose beliefs can be bought out for a few quid; and the giver, who becomes one who needs sugaring to be acceptable. Contradictory messages regarding the value of psychiatry are the last thing people with schizophrenia need from us, never mind our staff and the public.

David Ogden Locum Consultant in Old Age Psychiatry, Forest of Dean, email: david.ogden@ glos.nhs.uk

doi: 10.1192/pb.31.7.274a

\section{Quality assurance of training standards}

Professor Howard (Psychiatric Bulletin, February 2007, 31, 41-43) highlights the training standards set out by the Postgraduate Medical Education and Training Board (PMETB; http://www.pmetb. org.uk). One of these states that all trainees must attend a departmental induction, which includes information on the curriculum, their duties and reporting arrangements.

We conducted a survey of the existing senior house officer ( $\mathrm{SHO}$ ) induction programmes in the Eastern Deanery to assess if any changes are needed to fulfil PMETB standards. Each area has a trust and local induction, which varied in format and content. Some programmes run on consecutive days and others are incorporated into lunchtime educational meetings. They all consist of sessions on medical staffing, on-call arrangements and talks by pharmacy staff. Some trusts include all mandatory training such as cardiopulmonary resuscitation, fire safety, etc. Lectures on specific skills (e.g. psychiatric emergencies), a tour of the hospital sites including the library, and meeting with clinical tutors or educational supervisors are commonly included in the induction programmes. A SHO handbook was provided by a majority of trusts. Only one trust gave an introduction to the psychiatric curriculum.

The SHO feedback showed that the most useful part of an induction programme was meeting with other colleagues and receiving practical information, including details of on-call arrangements and contact numbers. They favoured shorter sessions run over several weeks.

This survey reflects the variability of $\mathrm{SHO}$ induction programmes within one deanery. Clear guidance is needed to ensure the standardisation and quality of the programme throughout a region.

Claire Dibben Specialist Registrar in General Adult Psychiatry, Kit Wa Chan Specialist Registrar in General Adult Psychiatry, $\quad$ *Carol Gregory Consultant Psychiatrist and Clinical Tutor, Fulbourn Hospital, Cambridge CB1 5EF, email: carol.gregory@ cambsmh.nhs.uk

doi: 10.1192/pb.31.7.274b

\section{Rebound hypertension following withdrawal of clonidine}

We report a 15-year-old girl with mild intellectual disability and Tourette's syndrome who also had features of hyperkinetic disorder. She had responded poorly to earlier trials of haloperidol and methylphenidate and was on $300 \mu \mathrm{g}$ clonidine twice a day, $2 \mathrm{mg}$ risperidone daily, $20 \mathrm{mg}$ citalopram daily and $2 \mathrm{mg}$ lorazepam a day. However, these medications were having minimal effects on her behaviour and her tics were also uncontrolled.

With no fixed protocol for clonidine withdrawal an enquiry was made to the hospital pharmacy and the manufacturer who suggested a withdrawal rate of $50 \mu \mathrm{g}$ every third day. A week after the withdrawal regimen she was admitted as an emergency to the children's ward with symptoms of blurred vision and high blood pressure. All investigations were normal except for elevated cholesterol and triglyceride levels.

A literature search did not yield any results for a safe rate of clonidine withdrawal to avoid the potentially dangerous side-effects of rebound hypertension in children. The manufacturer, Boehringer Ingelheim, informed us that there were no recommendations for withdrawing clonidine apart from the fact that it has to be withdrawn gradually.

Since clonidine is used in children and young people to treat tic and conduct disorders, sleep disturbances, posttraumatic stress disorder, developmental 